\title{
Article \\ Differential Expression of Genes Associated with Chromatin Modifications in Skeletal Muscle during Aerobic Training Program
}

\author{
Yecid Mina-Paz ${ }^{1, *(D)}$, Alejandra Rodríguez-Ortiz ${ }^{2}$ (D) Daniela Hernández-Pérez ${ }^{3,4}$, Julio César Montoya-Villegas ${ }^{4}$, \\ Adalberto Sánchez-Gómez ${ }^{4}$ and Felipe García-Vallejo ${ }^{4}$ \\ 1 Health and Movement Research Group, Faculty of Health, Universidad Santiago de Cali, \\ Cali 76001, Colombia \\ 2 SIT Consulting-Science, Innovation \& Technology, Cali 76001, Colombia; \\ alejandra.rodriguez@correounivalle.edu.co \\ 3 Faculty of Education and Sports Sciences, University Institution National Sports School, Cali 76001, Colombia; \\ daniela.hernandez.perez@correounivalle.edu.co \\ 4 Department of Physiological Sciences, School of Basic Sciences, Faculty of Health, University of Valle, \\ Cali 76001, Colombia; julio.montoya@correounivalle.edu.co (J.C.M.-V.); \\ adalberto.sanchez@correounivalle.edu.co (A.S.-G.); jesus.garcia@correounivalle.edu.co (F.G.-V.) \\ * Correspondence: yecid.mina@correounivalle.edu.co
}

check for

updates

Citation: Mina-Paz, Y.;

Rodríguez-Ortiz, A.; Hernández-Pérez,

D.; Montoya-Villegas, J.C.;

Sánchez-Gómez, A.; García-Vallejo, F.

Differential Expression of Genes

Associated with Chromatin

Modifications in Skeletal Muscle

during Aerobic Training Program.

Appl. Sci. 2022, 12, 1159. https://

doi.org/10.3390/app12031159

Academic Editor: Daniela Galli

Received: 17 November 2021

Accepted: 23 December 2021

Published: 23 January 2022

Publisher's Note: MDPI stays neutral with regard to jurisdictional claims in published maps and institutional affiliations.

Copyright: (c) 2022 by the authors. Licensee MDPI, Basel, Switzerland. This article is an open access article distributed under the terms and conditions of the Creative Commons Attribution (CC BY) license (https:// creativecommons.org/licenses/by/ $4.0 /)$.

\begin{abstract}
Introduction: Skeletal muscle plasticity in exercise can be modulated by epigenomic events such as gene silencing, histone modifications and DNA methylation. In this context, our objective was to analyze the expression of genes associated with chromatin modifications in human muscle biopsies of vastus lateralis after a 20 week training program. Methods: Using data from DNA microarray experiments registered in the NCBI GEO DataSet database GSE117070, we calculated the Z ratio values as the criterion to evaluate the differential expression of genes associated with chromatin modification during aerobic training in skeletal muscle. Using the web interface GENEMANIA, we built a co-expression interaction network with the overexpressed genes. We compared Z-score values obtained from pre-trained and post-trained samples through nonparametric tests. Results: We found 10 overexpressed genes after the 20 week training program, namely, EZH1, KMT2A, KMT2D, KDM4C, KDM6A, CREBBP, HDAC10, HDAC4, DNMT3L, and H2AX. The most relevant biological processes obtained from the network included chromatin organization (FDR $9.04 \times 10^{-9}$ ) and histone modification (FDR $9.04 \times 10^{-9}$ ). Conclusions: In skeletal muscle, after aerobic training, there is overexpression of genes associated with the modification of the chromatin through alterations in histones and DNA, resulting in epigenetic transcriptional changes.
\end{abstract}

Keywords: aerobic training; epigenomics; gene expression; chromatin modifications

\section{Introduction}

The human genome project gave fundamental knowledge to further our understanding of the genomic landscape and the relationship between genes and phenotypical traits [1] Sports performance mediated by advances in "omics" technologies and genomic sequencing has allowed new possibilities in the study of molecular mechanisms underlying physical conditions [2]. In fact, the molecular markers involved with performance and the manifestations of physical qualities, such as strength, flexibility, endurance, speed, and agility, can be useful in sports selection processes. This systemic approach involves the current functional state assessment of an athlete and the selection of sports specialization, among others [3].

The environmental modulation in skeletal muscle plasticity during exercise in healthy and/or sick people is mediated by the regulation of gene expression through epigenomic events such as histone modification and DNA methylation [4]. The physical performance 
of an athlete is built upon a series of factors such as lifestyle, training process, physiological, psychological, political, and social and genetic aspects. Since 2012, Sportomics, as a branch of metabolomics, has focused on understanding the modifications induced by exercise, through evaluations of signaling molecules during real or imitated conditions faced by athletes [5].

Oxygen concentrations vary as a function of the type and intensity of exercise, modifying cellular metabolism [6-9]. During aerobic or anaerobic stress, cellular metabolism has become an important biological node that governs cellular behavior specifically in skeletal muscle $[10,11]$. During physical activity or exercise, energy needs are met, providing chemical molecules that maintain homeostasis, proliferation, and cell function, through cardiorespiratory and metabolic function [12]. Acetyl-CoA, adenosine triphosphate (ATP), $\alpha$-ketoglutarate, and NAD/NADP, among other metabolites, facilitate changes in nutrient intake or availability through complete reprogramming of cellular metabolism $[13,14]$.

The metabolism of maximal oxygen uptake $\left(\mathrm{VO}_{2 \mathrm{max}}\right)$ is the result of interactions of biochemical reactions that absorb nutrients to process them and satisfy the demands during aerobic and/or anaerobic stress, such as energy generation and biosynthesis [15-18]. The intermediates of these metabolic reactions modify the epigenome [19] through cofactors and substrates and for different enzymes, allowing the signaling to the state of the nuclear chromatin of the changes derived from training.

Chromatin is a complex of DNA and basic proteins called histones, which are topologically arranged as a complex and discrete unit called the nucleosome [20]. Each nucleosome can condense itself, creating a tight barrier that constrains the easy access of transcriptional factors to the genome. Among the chemical modifications made to nucleosomal histones are methylation and acetylation.

That is why the understanding and interpretation of these changes allows an optimal development of sports planning involving all changes in histones in terms of $\mathrm{VO}_{2 \max }$ markers during aerobic training. In this context, our objective was to analyze the expression of genes related to chromatin modifications in human muscle biopsies of vastus lateralis after a 20 week training program, using a bioinformatics approach.

\section{Methodology}

Ninety-four genes associated with chromatin modification that were previously reported in the NCBI database of Gene Entrez. (https:/ / www.ncbi.nlm.nih.gov/gene (accessed on 17 May 2019); see Supplementary Table S1) were used for bioinformatic analysis. The transformed expression values of $\log _{2}$ intensity were obtained from a DNA microarray, which is freely accessible from the GEO database with registration code GSE117070. (https: / / www.ncbi.nlm.nih.gov/geo/ query / acc.cgi?acc=GSE117070 (accessed on 17 May 2019)) [21]. The selected experiment included 82 quadriceps (vastus lateralis) muscle samples from 90 Caucasians and 40 African Americans with gene expression data from more than 43,797 probes. Two categories were established, "descendants" between 17 and 40 years old and "parents" between 40 and 65 years old, who had not done training or any physical activity for the past 3 months prior the beginning of the study, and who did not suffer from any chronic noncommunicable disease. All individuals included in the study were assessed before and after the 20 week training program. The study did not include a control group, because it was found that there is an important difference in the application of training programs against the risk factors evaluated before and after. Hence, the participants themselves were the control and intervention groups. The gene expression profile was generated from muscle biopsies before and after the application of a training program.

As described in the study regarding the transcriptome, the training program that lasted 20 weeks had the following phases: three sessions of $30 \mathrm{~min}$ each, at $55 \%$ of $\mathrm{VO}_{2 \max }$ in the first 2 weeks; three sessions of 35 min each, at $55 \%$ of $\mathrm{VO}_{2 \max }$ for 2 weeks; three sessions of $35 \mathrm{~min}$ each, at $65 \%$ of $\mathrm{VO}_{2 \max }$ for 2 weeks; three sessions of $40 \mathrm{~min}$ each, at $65 \%$ of $\mathrm{VO}_{2 \max }$ for 2 weeks; three sessions of 40 min each, at $70 \%$ of $\mathrm{VO}_{2 \max }$ for 2 weeks; 
three sessions of $45 \mathrm{~min}$ each, at $70 \%$ of $\mathrm{VO}_{2 \max }$ for 2 weeks; three sessions of 45 min each, at $75 \%$ of $\mathrm{VO}_{2 \max }$ for 2 weeks; three sessions of 40 min each per week, at $75 \%$ of $\mathrm{VO}_{2 \max }$ for 6 weeks. Heart rate monitoring was continuous through a cycle ergometer [21]. For this study, we analyzed the expression of genes associated with chromatin modifications in skeletal muscle before and after the 20 week training program.

\section{Differential Gene Expression Quantification}

The experiment carried out by Bouchard (1995) was applied for gene expression profiles. The platform used was the GPL570 [HG-U133_Plus_2] Affymetrix Human Genome U133 Plus 2.0 Matrix. The U133 GeneChip genome probes were replicated identically on the U133 Plus 2.0 GeneChip human genome matrix. GenBank ${ }^{\circledR}$, dbEST, and RefSeq were the sequences used to derive the referenced probes. We used the $\log _{2}$ data obtained from the DNA microarray to analyze the gene expression by calculating the Z-score [22] according to Equation (1).

$$
Z-\text { score }=\frac{(\text { Log intensity of } G-\text { mean } \log \text { intensity } G \ldots G n)}{\text { Standard Deviation } \log G \ldots G n} .
$$

We normalized the Z-score values on a linear scale $-3.0 \leq 0 \geq+3.0$ ( $p$-value $<0.001$ ) and calculated the average values per gene for pre-training and post-training samples. With these data, we estimated the differential gene expression using the Z-ratio (Equation (2)). A value higher than 1.96 obtained by a gene was considered overexpression [22].

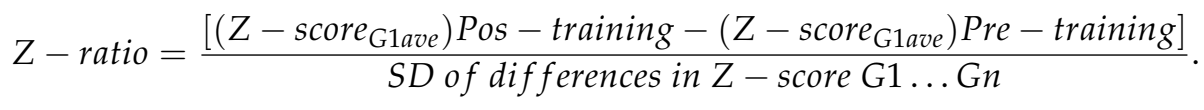

\section{Co-Expression Interaction Network}

With the overexpressed genes found in this study, a co-expression interaction network was made using GENEMANIA (https:/ / genemania.org/ (accessed on 17 September 2020)). The top 10 relevant protein functions associated with the genes was obtained from the network.

\section{Statistical Analysis}

We performed nonparametric analyses to compare the Z-score values between posttrained and pre-trained samples. We set the significance level to 0.05 . Moreover, we used a two-tailed $t$-test to calculate the statistical differences in the $\log _{2}$ values of pre-trained and post-trained samples. To classify the gene expression of the muscle biopsy samples under pre-training and post-training conditions, we conducted a principal component analysis (PCA) in SPSS program, version 25.

\section{Results}

Out of the 94 genes that were analyzed in the present study, seven of them were significantly overexpressed in the vastus lateralis muscle samples. As shown in Table 1, they corresponded to enhancer of zeste 1 polycomb repressive complex 2 subunit (EZH1), lysine methyltransferase 2A (KMT2A), lysine methyltransferase 2D (KMT2D), lysine demethylase 4C (KDM4C), lysine demethylase 6A (KDM6A), CREB-binding protein (CREBBP), histone deacetylase 10 (HDAC10), histone deacetylase 4 (HDAC4), DNA methyltransferase 3 like (DNMT3L), and $\mathrm{X}$ variant histone (H2AX). Thus, $10.6 \%$ of the 94 genes analyzed may explain the behavior of $\mathrm{VO}_{2}$ in relation to skeletal muscle activity, for the target population in this study. 
Table 1. Description and expression data of overexpressed genes associated with chromatin modifications included in the present study.

\begin{tabular}{cccccc}
\hline Gene ID & Symbol & Z-Score Post & Z-Score Pre & Z-Ratio & $p$-Value * \\
\hline 2145 & EZH1 & -0.03 & -0.19 & 2.15 & $<0.00001$ \\
4297 & KMT2A & -0.85 & -1.08 & 3.03 & $<0.00001$ \\
8085 & KMT2D & -0.98 & -1.15 & 2.30 & $<0.00001$ \\
23081 & KDM4C & 0.53 & 0.33 & 2.69 & $<0.00001$ \\
7403 & KDM6A & -0.84 & -1.11 & 3.64 & $<0.00001$ \\
1387 & CREBBP & -0.09 & -0.24 & 2.09 & $<0.00001$ \\
83933 & HDAC10 & 0.15 & -0.00 & 2.00 & $<0.00001$ \\
9759 & HDAC4 & -0.12 & -0.30 & 2.38 & $<0.00001$ \\
29947 & DNMT3L & -0.78 & -0.95 & 2.35 & $<0.00001$ \\
3014 & H2AFX & 0.33 & 0.12 & 2.83 & $<0.00001$ \\
\hline
\end{tabular}

*Statistically significant $p$-value.

The most significant biological processes associated with the overexpressed genes obtained from the constructed network included chromatin organization (FDR $9.04 \times 10^{-9}$ ) and histone modification (FDR $9.04 \times 10^{-9}$ (Table 2), which are relevant for the function and contraction of muscles.

Table 2. Description of outstanding biological processes obtained from the network constructed with the genes significantly associated with chromatin modifications in vastus lateralis muscle.

\begin{tabular}{cccc}
\hline GO_ID & Description & Gene Count & FDR \\
\hline 6325 & Chromatin organization & 9 & $9.04 \times 10^{-9}$ \\
16570 & Histone modification & 8 & $9.04 \times 10^{-9}$ \\
18205 & Peptidyl-lysine modification & 7 & $1.07 \times 10^{-7}$ \\
16571 & Histone methylation & 5 & $2.04 \times 10^{-6}$ \\
43414 & Macromolecule methylation & 6 & $2.32 \times 10^{-6}$ \\
6996 & Organelle organization & 10 & $4.69 \times 10^{-5}$ \\
40029 & Regulation of gene expression, epigenetic & 5 & $4.69 \times 10^{-5}$ \\
34968 & Histone lysine methylation & 4 & $5.13 \times 10^{-5}$ \\
10604 & Positive regulation of macromolecule metabolic process & 10 & $5.25 \times 10^{-5}$ \\
45934 & Negative regulation of nucleobase-containing compound metabolic process & 8 & $6.02 \times 10^{-5}$ \\
51172 & Negative regulation of nitrogen compound metabolic process & 9 & $6.51 \times 10^{-5}$ \\
\hline
\end{tabular}

The significant protein functions associated with the genes assessed, obtained from the co-expression network, were methyltransferase complex and deacetylase activity in 20 weeks of aerobic training (Figure 1 ).

The PCA showed that $53 \%$ and $54 \%$ of the accumulated variance was attained within two components in the pre-training (Figure 2) and post-training samples (Figure 3), respectively. 


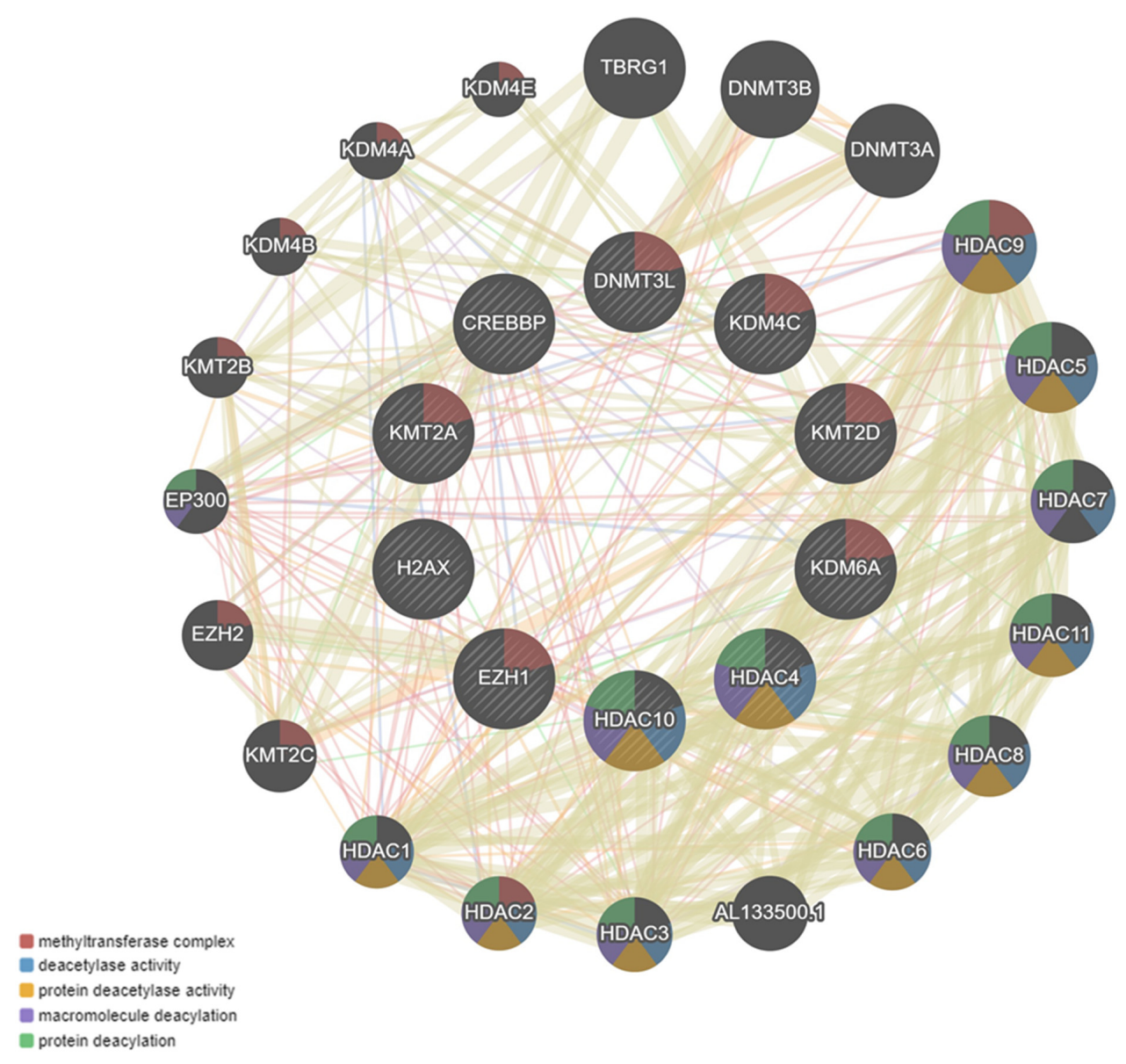

Figure 1. Co-expression interaction network obtained with the 10 significantly overexpressed genes associated with muscle function evaluated.



Figure 2. Principal component analysis of pre-training samples with the overexpressed genes found in this study shown as green dots. 


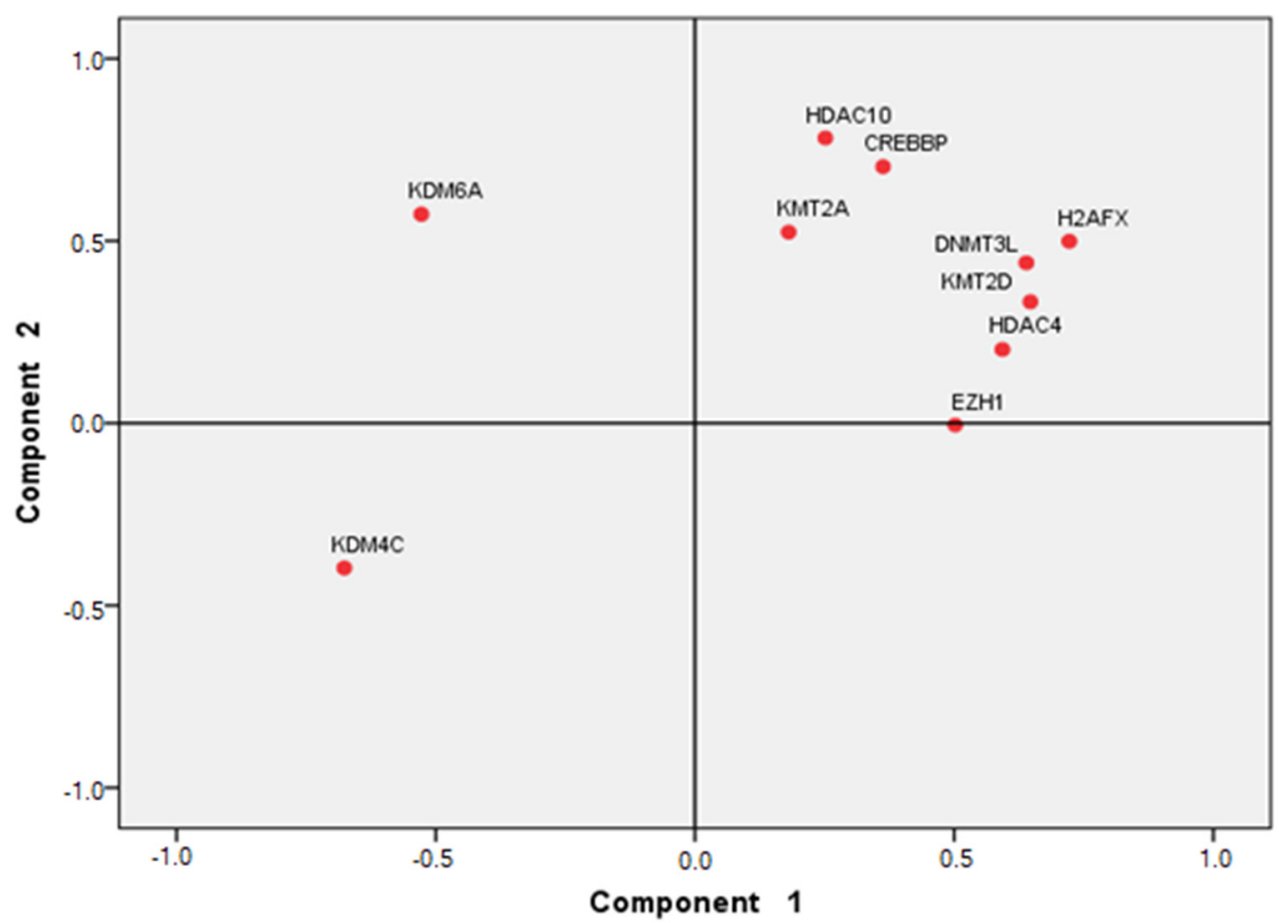

Figure 3. Principal component analysis of post-training samples with the overexpressed genes found in this study shown as red dots.

\section{Discussion and Conclusions}

In the present study, our objective was to perform a bioinformatic analysis of the differential expression of genes in muscle associated with chromatin modification from biopsy samples of the vastus lateralis of the quadriceps muscle in humans who were included in a 20 week aerobic exercise program. The original study that provided the source data for this analysis was presented by Bouchard in 1995, the HERITAGE family study, which presented the methodology describing the role of the genotype in the cardiovascular, metabolic, and hormonal responses to aerobic exercise training, in 90 Caucasian families and 40 African American families with both parents and three or more biological adult descendants [21]. On the contrary, in our article, we did not focus the objectives, design, and measurement protocol. Moreover, in the study by Clarke et al. [23], which used the same source data, the authors found that knockdown of the mTOR-independent factor Eif6, which we predicted to be a key regulator of this process, affects mitochondrial respiration efficiency, ROS production, and exercise performance. Conversely, our results were focused on the genes associated with chromatin changes due to aerobic training.

The overexpressed genes found in the present study corresponded to EZH1, KMT2A, KMT2D, KDM4C, KDM6A, CREBBP, HDAC10, HDAC4, DNMT3L, and H2AX. All of them were previously reported to be important in chromatin organization and histone modification, including histone methylation in skeletal muscle described by the biological processes in Table 2, allowing changes in the sarcomere specifically in the thin actin filament and reshaping the cell to a type of muscle fiber that adjusts to the needs of training, as explained by Mina-Paz et al. [24]. For this reason, any dysregulation of these genes can affect their own functions and those of associated genes.

Our results correlate with the study by McGee et al. [25] which analyzed the impact of exercise on global histone modifications leading to transcriptional activation and chromatin remodeling in human skeletal muscle samples. The authors found that, during exercise, two histone deacetylase activity (HDAC) proteins were exported from the nucleus of the cells, not allowing them to accomplish their function as transcriptional repressors. In addition, Lim et al. [26] studied the responses of histone alterations to resistance exercise in skeletal muscle in relation to resistance training; they found a significant upregulation 
of the distribution of acetylated histone 3 (H3Acet3), monomethylated $\mathrm{H} 3$ in lysine 4 (H3threeMetK4), and trimethylated in lysine 27 (H3-threeMetK27). However, in our study, we found overexpression of lysine methyltransferase KMT2A and KMT2D, which are mainly responsible for the methyltransferase activity in lysine 4 of histone H3 (H3MetK4).

During muscle adaptations to resistance or aerobic training, the isoforms of myofibrillar proteins most frequently change in order to facilitate the entry of calcium during muscle relaxation; among these proteins are the heavy chain of myosin (MYH), the thin actin filaments, the troponin/tropomyosin complex, and even proteins of the sarcoplasmic and mitochondrial reticulum $[27,28]$. Previous reports showed the exercise-induced transcription of GLUT4 in skeletal muscle through the deactivation of HDAC4/5, whereby suppressing the activity of HDAC4 led to an increase in the expression of GLUT4 during resistance training that started at $75 \%$ of $\mathrm{VO}_{2 \text { max }}$; however, in our study, resistance training started at $55 \%$ of $\mathrm{VO}_{2 \max }$, implicating the overexpression of HDAC4 and HDAC10. For this reason, glucose that enters through GLUT becomes the source of energy during resistance exercise, modifying performance and metabolic flow through intensity and total workload.

The overexpression of HDAC4 and HDAC10 in our study may be associated with metabolic-type enzymatic processes, which normally occur in the cytoplasm or mitochondria; the enzymes involved are pyruvate dehydrogenase complex (PDHC), lactate dehydrogenase (LHD), alanine aminotransferase (ALT), and succinate dehydrogenase (SDH) [29]. These enzymes supply metabolites that regulate the activity of chromatin-modifying or transcriptional regulatory enzymes to regulate gene expression and chromatin structure. One of those metabolic products is lactate; this behaves as an endogenous inhibitory metabolite of HDAC that targets the histone 4 complex [30], acetylating histone marks and inducing the overexpression found in this study. This increase in lactate concentration occurs during training when the athlete is subjected to an incremental test measured by oxygen consumption [31].

The changes presented in the training program induced the overexpression of enzymes such as DNMT3L that regulates CpG methylation and enzymes such as EZH1 that modulate trimethylation at lysine residues 4,27 , and 36 of histone H3. During this type of training program, the availability of the ATP used increases, in association with the changes generated in the mitochondria by the expression of PGC1 $\alpha$. Enzymes such as methionine adenosyl transferase (MAT) use ATP during methylation reactions [32], which are carried out by enzymes that regulate the methylation marks of DNA in lysine residues of histones and $\mathrm{CpG}$ islands.

The training program established for this study, with incremental intensity from $55 \%$ to $75 \%$ of $\mathrm{VO}_{2 \max }$, provides solid evidence supporting the hypothesis that, in skeletal muscle, there is overexpression of genes associated with the modification of chromatin through alterations in histones and DNA, resulting in epigenetic transcriptional changes.

Supplementary Materials: The following are available online at https:/ /www.mdpi.com/article/ 10.3390/app12031159/s1: Table S1. Description of overexpressed genes associated with chromatin modifications in skeletal muscle included in the present study.

Author Contributions: Y.M.-P., F.G.-V. and A.R.-O. performed data acquisition and information processing; Y.M.-P., F.G.-V., A.S.-G. and J.C.M.-V. wrote the introduction and the methods; Y.M.-P., F.G.-V. and D.H.-P. wrote the results, discussion, and conclusions. All authors have read and agreed to the published version of the manuscript.

Funding: The APC for this research was funded by the Directorate General of Research (Dirección General de Investigaciones) of the Universidad Santiago de Cali. This research has been funded by the vice-rectory of research at the Universidad del Valle through the scholarship 119-2019.

Institutional Review Board Statement: Not applicable.

Informed Consent Statement: Not applicable. 
Data Availability Statement: DNA microarray experiment whose registration code in the GEO database is GSE117070. (https:/ /www.ncbi.nlm.nih.gov/geo/query/acc.cgi?acc=GSE117070 accessed on 17 May 2019).

Acknowledgments: To the Universidad Santiago de Cali and the Universidad del Valle.

Conflicts of Interest: None of the authors presents a conflict of interest.

\section{References}

1. Collins, F.S.; Lander, E.S.; Rogers, J.; Waterston, R.H.; Conso, I.H. Finishing the euchromatic sequence of the human genome. Nature 2004, 431, 931-945. [CrossRef]

2. Guth, L.M.; Roth, S.M. Genetic influence on athletic performance. Curr. Opin. Pediatr. 2013, 25, 653. [CrossRef]

3. Malsagova, K.A.; Butkova, T.V.; Kopylov, A.T.; Izotov, A.A.; Rudnev, V.R.; Klyuchnikov, M.S.; Kaysheva, A.L. Molecular Portrait of an Athlete. Diagnostics 2021, 11, 1095. [CrossRef]

4. $\quad$ Rowlands, D.S.; Page, R.A.; Sukala, W.R.; Giri, M.; Ghimbovschi, S.D.; Hayat, I.; Cheema, B.S.; Lys, I.; Leikis, M.; Sheard, P.W.; et al. Multi-omic integrated networks connect DNA methylation and miRNA with skeletal muscle plasticity to chronic exercise in Type 2 diabetic obesity. Physiol. Genom. 2014, 46, 747-765. [CrossRef]

5. Coelho, W.S.; Viveiros de Castro, L.; Deane, E.; Magno-França, A.; Bassini, A.; Cameron, L.C. Investigating the Cellular and Metabolic Responses of World-Class Canoeists Training: A Sportomics Approach. Nutrients 2016, 8, 719. [CrossRef] [PubMed]

6. Hong, J.; Kim, K.; Kim, J.H.; Park, Y. The role of endoplasmic reticulum stress in cardiovascular disease and exercise. Int. J. Vasc. Med. 2017, 2017, 2049217. [CrossRef] [PubMed]

7. Antunes-Correa, L.M. Maximal oxygen uptake: New and more accurate predictive equation. Eur. J. Prev. Cardiol. 2018, 25, 1075-1076. [CrossRef]

8. Braunwald, E. Control of myocardial oxygen consumption: Physiologic and clinical considerations. Am. J. Cardiol. 1971, 27, 416-432. [CrossRef]

9. Cabrera-Hernandez, M.A.; Tafur-Tascon, L.J.; Cohen, D.D.; García-Corzo, S.A.; Quiñonez-Sánchez, A.; Povea-Combariza, C.; Tejada-Rojas, C.X. Concordance between the indirect VO2max value estimated through the distance in Yo-Yo intermittent recovery test level 1 and the direct measurement during a treadmill protocol test in elite youth soccer players. J. Hum. Sport Exerc. 2018, 13, S401-S412. [CrossRef]

10. Stong, L.; Haile, L.; Beyer, K.; Andreacci, J. Effect of Test Sequence on Maximal Anaerobic and Aerobic Power Achievements in Adults. Int. J. Exerc. Sci. 2021, 14, 657-665.

11. Bar-Or, O. The Wingate anaerobic test an update on methodology, reliability and validity. Sports Med. 1987, 4, 381-394. [CrossRef] [PubMed]

12. Thirumalai, T.; Therasa, S.V.; Elumalai, E.K.; David, E. Intense and exhaustive exercise induce oxidative stress in skeletal muscle. Asian Pac. J. Trop. Dis. 2011, 1, 63-66. [CrossRef]

13. Fisher-Wellman, K.; Bloomer, R.J. Acute exercise and oxidative stress: A 30 year history. Dyn. Med. 2009, 8, 1. [CrossRef] [PubMed]

14. Yucel, N.; Wang, Y.X.; Mai, T.; Porpiglia, E.; Lund, P.J.; Markov, G.; Garcia, B.A.; Bendall, S.C.; Angelo, M.; Blau, H.M. Glucose metabolism drives histone acetylation landscape transitions that dictate muscle stem cell function. Cell Rep. 2019, 27, 3939-3955. [CrossRef] [PubMed]

15. Chandel, N.S. Navigating Metabolism; Cold Spring Harbor Laboratory Press: Cold Spring Harbor, NY, USA, 2015 ; Chapter 1; pp. 1-6.

16. Plowman, S.A.; Smith, D.L. Exercise Physiology for Health, Fitness, and Performance, 4th ed.; Lippincott Williams \& Wilkins: Philadelphia, PA, USA, 2014; pp. 55-58.

17. Mondal, H.; Mishra, S. Effect of BMI, Body Fat Percentage and Fat Free Mass on Maximal Oxygen Consumption in Healthy Young Adults. J. Clin. Diagn. Res. 2017, 11, CC17-CC20. [CrossRef]

18. Treacher, D.F.; Leach, R.M. Oxygen Transport-1. Basic principles. BMJ 1998, 317, 1302-1306. [CrossRef]

19. Reid, M.A.; Dai, Z.; Locasale, J.W. The impact of cellular metabolism on chromatin dynamics and epigenetics. Nat. Cell Biol. 2017, 19, 1298-1306. [CrossRef]

20. Felsenfeld, G.; Groudine, M. Controlling the double helix. Nature 2003, 421, 448-453. [CrossRef]

21. Bouchard, C.L. The heritage family study. Aims, design, and measurement protocol. Med. Sci. Sports Exerc. 1995, 27, 721-729. [CrossRef]

22. Cheadle, C.; Vawter, M.P.; Freed, W.J.; Becker, K.G. Analysis of microarray data using Z score transformation. J. Mol. Diagn. 2003, 5, 73-81. [CrossRef]

23. Clarke, K.; Ricciardi, S.; Pearson, T.; Bharudin, I.; Davidsen, P.K.; Bonomo, M.; Brina, D.; Scagliola, A.; Simpson, D.M.; Beynon, R.J.; et al. The Role of Eif6 in Skeletal Muscle Homeostasis Revealed by Endurance Training Co-expression Networks. Cell Rep. 2017, 21, 1507-1520. [CrossRef] [PubMed]

24. Mina-Paz, Y.; Zambrano, D.C.; Matta, A.J.; Rodríguez, A.; Garcia-Vallejo, F. Muscle genomics and aerobic training. J. Hum. Sport Exerc. 2021, 17. in press. [CrossRef]

25. McGee, S.L.; Fairlie, E.; Garnham, A.P.; Hargreaves, M. Exercise-induced histone modifications in human skeletal muscle. J. Physiol. 2009, 587, 5951-5958. [CrossRef] [PubMed] 
26. Lim, C.; Shimizu, J.; Kawano, F.; Kim, H.J.; Kim, C.K. Adaptive responses of histone modifications to resistance exercise in human skeletal muscle. PLoS ONE 2020, 15, e0231321. [CrossRef] [PubMed]

27. Egan, B.; Zierath, J.R. Exercise metabolism and the molecular regulation of skeletal muscle adaptation. Cell Metab. 2013, 17, 162-184. [CrossRef]

28. Niu, Y.; Wang, T.; Liu, S.; Yuan, H.; Li, H.; Fu, L. Exercise-induced GLUT4 transcription via inactivation of HDAC4/5 in mouse

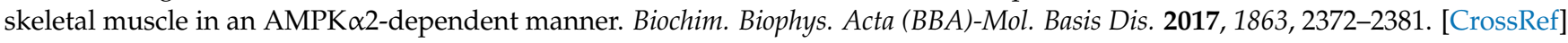

29. Boukouris, A.E.; Zervopoulos, S.D.; Michelakis, E.D. Metabolic enzymes moonlighting in the nucleus: Metabolic regulation of gene transcription. Trends Biochem. Sci. 2016, 41, 712-730. [CrossRef]

30. Latham, T.; Mackay, L.; Sproul, D.; Karim, M.; Culley, J.; Harrison, D.; Hayward, L.; Langridge-Smith, P.; Gilbert, N.; Ramsahoye, B. Lactate, a product of glycolytic metabolism, inhibits histone deacetylase activity and promotes changes in gene expression. Nucleic Acids Res. 2012, 40, 4794-4803. [CrossRef]

31. Bertuzzi, R.; Nascimento, E.M.; Urso, R.P.; Damasceno, M.; Lima-Silva, A.E. Energy system contributions during incremental exercise test. J. Sports Sci. Med. 2013, 12, 454-460.

32. Luka, Z.; Mudd, S.; Wagner, C. Glycine N-methyltransferase and regulation of S-adenosylmethionine levels. J. Biol. Chem. 2009, 284, 22507-22511. [CrossRef] 